\title{
Structure, Electrical Resistivity and Superconductivity of Low- alloyed $\gamma$-U Phase Retained to Low Temperatures by Means of Rapid Cooling
}

\author{
M. Krupska' ${ }^{1}$ N.-T. H. Kim-Ngan' ${ }^{1}$ S. Sowa ${ }^{1} \cdot$ M. Paukov ${ }^{2}$ I. Tkach $^{2}$ - D. Drozdenko ${ }^{2}$ L. Havela H $^{2}$ \\ Z. Tarnawski ${ }^{3}$
}

Received: 11 November 2015/Revised: 23 December 2015/Published online: 16 March 2016

(c) The Chinese Society for Metals and Springer-Verlag Berlin Heidelberg 2016

\begin{abstract}
The $\gamma-\mathrm{U}$ phase alloys can be retained down to low temperatures with less required alloying concentration by using the splat-cooling technique with a cooling rate better than $10^{6} \mathrm{~K} / \mathrm{s}$. Doping with 15 at.\% Mo, Pt, Pd, Nb leads to a stabilization of the cubic $\gamma$-U phase, while it requires much higher $\mathrm{Zr}$ concentrations ( $\geq 30$ at. $\% \mathrm{Zr}$ ). All $\mathrm{U}-T$ splats become superconducting with $T_{\mathrm{c}}$ in the range of 0.61-2.11 K. A good agreement of the experimentally observed specific-heat jump at $T_{\mathrm{c}}$ with that from BCS theory prediction was obtained for U-15 at.\% Mo consisting of the $\gamma-\mathrm{U}$ phase with an ideal $b c c$ A2 structure.
\end{abstract}

KEY WORDS: Crystal structure; Electrical resistivity; Metallic system; Superconductivity

\section{Introduction}

\subsection{Cubic $\gamma$-U Phase}

Uranium $(Z=92)$ is a main component of the most nuclear fuels. Fundamental physical properties of elemental uranium have been investigated thoroughly for the orthorhombic $\alpha$-U phase (space group Cmcm) [1, 2], since only this phase is stable at and below room temperature. The superconductivity of (natural) uranium was first discovered at $1.3 \mathrm{~K}$ in 1942 [3]. Most recent data have reported $T_{\mathrm{c}}=0.78 \mathrm{~K}[4,5]$. However, no signature of the

Available online at http://link.springer.com/journal/40195

N.-T. H. Kim-Ngan

tarnawsk@up.krakow.pl

1 Institute of Physics, Pedagogical University, Podchorazych 2, 30084 Kraków, Poland

2 Faculty of Mathematics and Physics, Charles University, Ke Karlovu 5, 12116 Prague, Czech Republic

3 Faculty of Physics and Applied Computer Science, AGH University of Science and Technology, 30-059 Kraków, Poland superconductivity was found down to $0.02 \mathrm{~K}$ at ambient pressure in good-quality single crystals of uranium, although the charge-density-wave (CDW) states [5] were found to be developed fully at low temperatures in those crystalline uranium specimens [6].

The $\gamma$-U phase with a body-centered cubic structure (space group $\operatorname{Im} \overline{3} m$ ) is stable at high temperatures between $(1049 \pm 2)$ and $1408 \mathrm{~K}[1,2]$. It can be retained to the room temperature by alloying with $\mathrm{Zr}, \mathrm{Nb}, \mathrm{Mo}, \mathrm{Pd}$, Pt, etc. [7]. Mo has a large solubility in $U(\approx 35$ at.\%) and thus is considered as a good candidate to stabilize $\gamma$-U. For instance, the single-phase $\gamma$-U alloy has been reported for U-8 wt\% Mo (U-16.5 at.\% Mo) under normal furnace cooling conditions [8].

The large interest in stabilization of $\gamma-\mathrm{U}$ phase alloys has mostly come from the viewpoint of metallurgy. In the late 1970s, massive research programs were launched in the USA to develop the low enriched uranium (LEU, $<20 \%{ }^{235} \mathrm{U}$ ) fuels $[9,10]$. The research showed that the $\gamma$-U-Mo alloys were the most promising candidates for LEU fuels; e.g., they have a higher stability under irradiation and they are more resistant against swelling (than $\alpha$-U alloys) [11, 12]. Besides, they are much more stable phase for manufacture and greater fissile load [13]. U-Mo alloys were also found to be the top 
performers among many U-based fuel alloys in the screening tests. U-10 wt\% Mo was selected for the US reactors, while many European reactors continue to use the U-7 Mo [10]. This percent $(7-10 \mathrm{wt} \% \mathrm{Mo})$ in the metallic $\mathrm{U}$ is enough to obtain the $\gamma$-U phase stability.

\subsection{Use of Ultrafast Cooling in Stabilizing the Cubic $\gamma$-U Phase}

The $5 f$ electronic states in many uranium-based compounds are generally close to the verge of localization, which brings up fascinating many-body physics from the fundamental research viewpoint. In this respect, it is necessary to determine the basic thermodynamic properties of the lessknown $\gamma$-U phase alloys. Except of old reports from 1960s on the superconductivity of the $\gamma$-U phase around $2 \mathrm{~K}$ in water-quenched U-Mo and $\mathrm{U}-\mathrm{Nb}$ alloys [14, 15], no more detailed data exist for the $\gamma-\mathrm{U}$ alloys.

We have been interested in stabilization of $\gamma-\mathrm{U}$ alloys and characterization of their fundamental electronic properties, especially their superconductivity. It was shown earlier that the rapid quenching (with a cooling rate of about $10^{5} \mathrm{~K} / \mathrm{s}$ ) of certain alloys from the melting point could lead to a formation of new metastable phases and/or amorphous solid phases [16]. Indeed, the splat-cooling technique has been used for searching novel microstructure or amorphous uranium [17]. Recently, using ultrafast cooling from the melt to room temperature, we can retain the cubic $\gamma$-U phase in $\mathrm{U}_{-}$ $T$ alloys. In our equipment, the molten metal is dropped between two colliding massive copper anvils, yielding a cooling rate better than $10^{6} \mathrm{~K} / \mathrm{s}$ [18]. Another advantage of this technique is that it can work with small amounts of material (200-300 mg). We can then proceed with characterization of low-temperature properties of the $\gamma$-U phase alloys. Starting with Mo alloying, we succeeded to suppress the $\gamma$-U phase with about 11 at.\% Mo $[18,19]$.

In the present work, we concentrate on stabilization of the $\gamma$ $\mathrm{U}$ phase by a combination of ultrafast cooling and other $T$ alloying ( $T=\mathrm{Pt}, \mathrm{Pd}, \mathrm{Nb}, \mathrm{Zr}$ ). We have also investigated the low-temperature properties of the obtained splat-cooled U$T$ alloys, in particular their superconductivity. Some results are compared with those obtained earlier for U-Mo splats.

\section{Experimental}

U- $T$ alloys ( $T=\mathrm{Mo}, \mathrm{Pt}, \mathrm{Nb}, \mathrm{Zr}$ ) with low- $T$ contents (up to 30 at.\%) were prepared using natural $\mathrm{U}$ ( $2 \mathrm{~N} 8$ purity or better) and $T$ element (3N 8 or better) by arc melting on a copper plate in argon atmosphere. The splat-cooled sample was prepared from the alloy ingot by splat-cooling technique (using the HV splat cooler from Vakuum Praha) and had a shape of irregular disk with a diameter of approx.
$20 \mathrm{~mm}$ and a thickness of 100-200 $\mu \mathrm{m}$, as shown in Fig. 1 . More details of preparation of the splats have been reported earlier [18]. Throughout our work, the $T$ content is given in the atomic percent (at.\%).

The crystal structure of the splat-cooled alloys (splats) was investigated by X-ray diffraction (XRD) using the Bruker D8 Advance diffractometer with $\mathrm{Cu}-K \alpha$ radiation. The resistivity and specific heat measurements were carried out in the temperature range $0.3-300 \mathrm{~K}$ by means of standard techniques, such as closed-cycle refrigerator system (CCR) and Quantum Design Physical Properties Measurement System (PPMS) described earlier [18]. For investigations around the superconducting transitions, we performed those measurements in applied magnetic fields up to $7 \mathrm{~T}$. Additional phase purity analysis was performed by scanning electron microscope (SEM) equipped with an energy dispersive X-ray (EDX) analyzer. The splats show a homogeneous distribution of the alloying elements with concentrations corresponding to the nominal ones. Some $\mathrm{C}, \mathrm{O}$ and $\mathrm{Cu}$ contamination was observed for the splats after preparation. $\mathrm{Cu}$ on the surface comes evidently from the $\mathrm{Cu}$ anvils of the splat cooler. Also, $\mathrm{C}$ and $\mathrm{O}$ are related to the surface (resulting from segregation) [19]. Electron backscattering diffraction (EBSD) analysis has been employed to study the microstructure and the texture of several splats (next section).

\section{Results and Discussion}

\subsection{Stabilization of the Cubic $\gamma$-U Phase in U-Mo Splats}

We have investigated thoroughly the crystal structure of U-Mo splats with Mo concentration of 0 (pure U splat), 1,

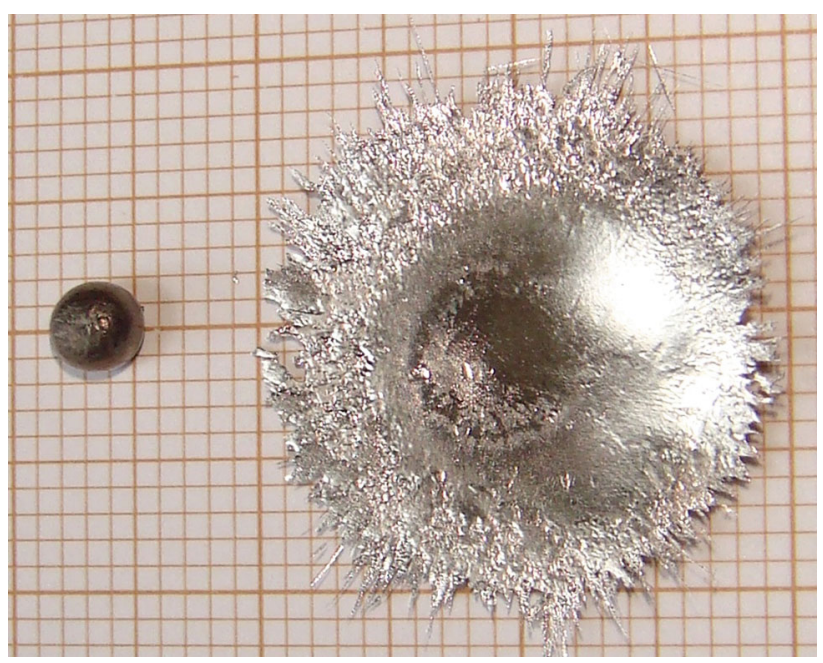

Fig. 1 Photograph of the bulk ingot with a mass of $\approx 300 \mathrm{mg}$ (left) prepared by the arc furnace and the resulting splat-cooled sample disk (right) produced by HV splat cooler 
$2,4,6,10,11,12,13,15,17$ at.\%, in order to determine precisely the minimal Mo concentration necessary for obtaining pure cubic $\gamma-\mathrm{U}$ phase. Details of our investigations of crystal structure and phase stability have been reported earlier [18, 19]. For a necessity of a comparison with other U-based splats, we summarize briefly the main outcome obtained on U-Mo splats below:

- The orthorhombic $\alpha$-U phase disappeared, and the $\gamma-\mathrm{U}$ phase developed fully in the alloys with $\geq 11$ at.\% Mo. A pure cubic $\gamma-\mathrm{U}$ phase without any distortion or diffusion is revealed only for U-15 at.\% Mo (and also for U-17 at.\% Mo) (see Fig. 1 in Ref. [19]).

- The stable $\gamma$-U alloys were obtained in the as-formed state without any additional sample treatment. Moreover, no aging or phase transformation/decomposition was observed when exposed to air. They show even very good resistance against any hydrogen absorption in the hydrogen atmosphere with the pressure below $2.5 \times 10^{5} \mathrm{~Pa}(2.5$ bar $)$.

The effect of the splat cooling can be seen in a better capability in retaining the $b c c$ type of structure for lower (by several at.\%) Mo concentrations. The reason can be, besides preventing the $\gamma-\alpha$ transformation, also avoiding the inhomogeneity of the Mo distribution in the $\gamma$-phase, which may appear due to the narrow bi-phase region between liquidus and $\gamma$-U with Mo. On the other hand, the splat cooling does not affect crystallinity: The materials do not turn amorphous.

EBSD analysis has been performed on several U-Mo splats, since this technique can give the direct information about spatial distribution of the phases and orientation of the grains. For this technique, a high surface quality is a prerequisite and special procedures have to be applied because of the tendency to oxidize rapidly in air [20]. In general, the first step of the sample preparation for all methods is mechanical polishing. The specimens were mechanically polished on $\mathrm{SiC}$ grinding papers (up to 4000 grid) using water and then coarse polished with using 3 and $1-\mu \mathrm{m}$ diamond abrasive on a nylon lap with a diamond suspension. To reveal the microstructure of the sample surface and to remove the surface oxide as the last step of preparation, Ar ion milling in the precision ion polishing system (PIPS) was performed. As an alternative technique, in situ focused ion beam (FIB) milling in chamber of SEM could be employed. Our experience shows that ion milling is a convenient way for preparing U-based surfaces. However, the surface of the specimen after the preparation procedure depends strongly on the sample compositions. Namely, the properties of $\gamma$-U surface (e.g., with more Mo doping) are very different from those of mostly $\alpha$-U ones which was found in low-doped $U$ alloys. Therefore, the preparation method should be still optimized for different
U alloys. Earlier published EBSD results for pure $\mathrm{U}$ and U-15 at.\% Mo splats [18] corroborated the XRD data. We succeeded to get also good EBSD map for other $\gamma-\mathrm{U}(\mathrm{Mo})$ alloys such as U-12 at.\% Mo (Fig. 2), revealing an equigranular grain structure without twinning and preferred crystallographic texture. The grain size is similar to that in U-15 at.\% Mo alloy (between 3.1 and $5.2 \mu \mathrm{m}$ ). We notice here that the XRD data revealed the $\gamma^{0}-\mathrm{U}$ phase in this splat. But the equipment cannot resolve such a small structure variation, i.e., a small tetragonal distortion of the $\gamma$-U phase. Therefore, the structure was identified as the $\gamma$ $\mathrm{U}$ phase. The important point is that for this splat, no any evidence for $\alpha$ - or $\alpha$-U related phases was observed in EBSD map, confirming the XRD data. Some isolated areas (shown as a black area in Fig. 2) were identified to be UC, confirmed by the Kikuchi pattern. Despite of our strong effort and a hard work on lower doped Mo alloys, no good EBSD map has been yet obtained, since it was difficult to get the free-oxidized surface which could diffract. The EBSD mapping on low Mo doping and other T-doping alloys is in the progress.

\subsection{Crystal Structure of U-T Alloy Splats $(T=\mathrm{Pt}, \mathrm{Pd}, \mathrm{Nb}, \mathrm{Zr})$}

We have extended our studies to the splat-cooled U-based alloys with other $T$ metals. We note here that while it is quite easy to produce the splats of $\mathrm{U}-\mathrm{Mo}, \mathrm{U}-\mathrm{Nb}$ and $\mathrm{U}-\mathrm{Zr}$ alloys in a wide range of $\mathrm{Mo} / \mathrm{Nb} / \mathrm{Zr}$ content, the similar procedure applied on U-Pd and U-Pt alloys turned out trickier. Namely, it is quite difficult to produce the splat disk for the Pd and Pt concentrations exceeding 5 at.\% [21]. Our investigations revealed that the XRD patterns, the resistivity drops and $T_{\mathrm{c}}$ values are very similar for the same alloying level whether Pt or Pd is used. Thus, we focus our attention on the U-Pt splats, trying to get a larger set of the splats with different $\mathrm{Pt}$ concentrations $(1,2,5,10$ and 15 at.\%), while only two Pd-containing splats were investigated for comparison.

The XRD patterns of U-Pt splats in the as-formed state are shown in Fig. 3a. For an easier comparison, we display normalized XRD patterns; i.e., the intensity of the most intense reflection around $36^{\circ}-37^{\circ}(=2 \theta)$ was set to 1 . The patterns are then shifted upwards to provide a better visual comparison. Increasing the $\mathrm{Pt}$ concentration leads to merging of several peaks around $36^{\circ}$, suppression of the low-index $\alpha$-peaks, vanishing of the high-index $\alpha$-peaks and a development of $\gamma$-peaks. The situation is very similar to U-Mo alloys, showing a coexistence of both $\alpha$ - and $\gamma-\mathrm{U}$ phases for splats with less than 10 at.\% alloying level. For both U-Pt and U-Pd splats, additional peaks appeared at around $37.6^{\circ}$ and $38.9^{\circ}$, i.e., between the $\alpha(002)$ and $\alpha(111)$ peaks. Such peaks are more visible for the Pt than Pd case. 
(a)

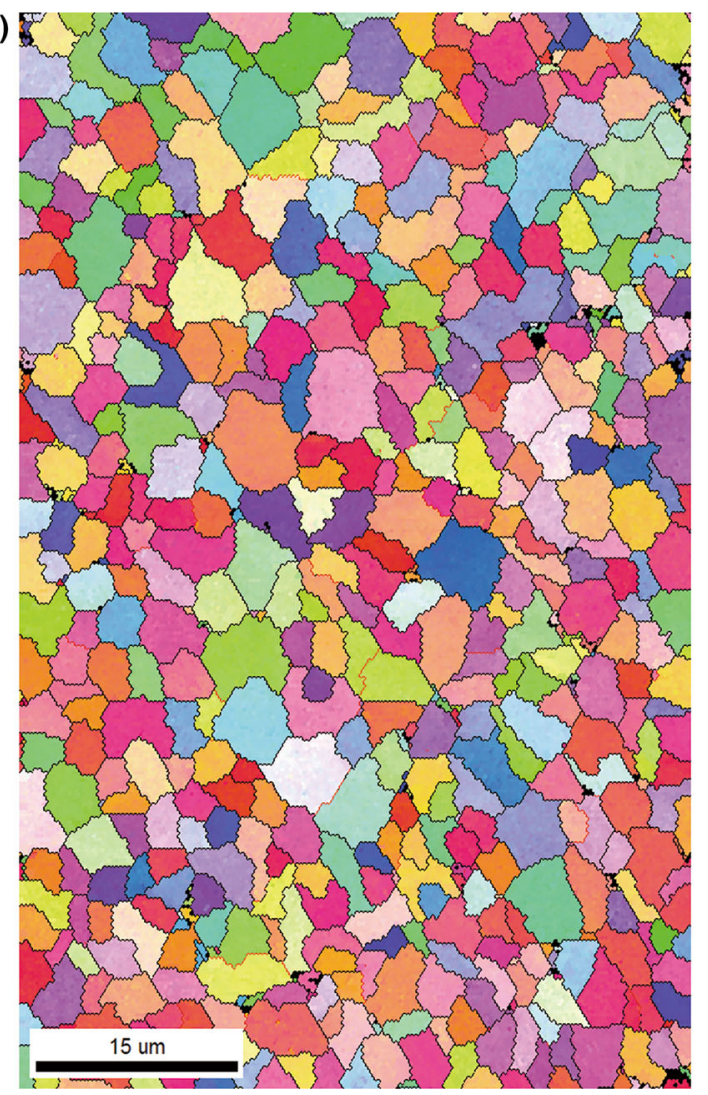

(b)

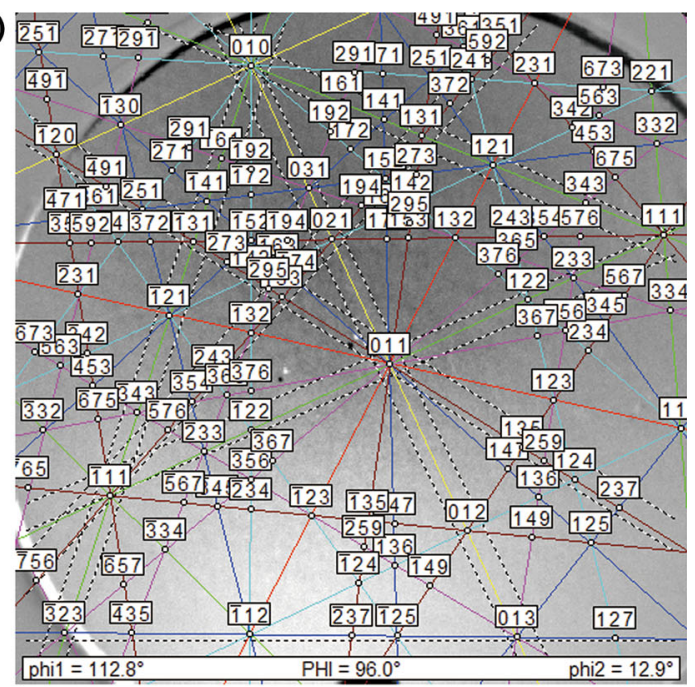

Fig. 2 EBSD crystallographic orientation map a and the Kikuchi pattern b highlighting the surface microstructure of the splat-cooled U-12 at.\% Mo alloy which identified only $\gamma$-U phase and UC (small black areas) with no evidence for $\alpha$-U or $\alpha$-U-related phases

Their intensity decreases with further increasing $\mathrm{Pt} / \mathrm{Pd}$ concentration, and they vanish in alloys with $\geq 10$ at.\% Pt/ $\mathrm{Pd}$. We cannot be conclusive on the basis of XRD about the spurious phase; we can only rule out the $\beta$-U phase, which has been never found in any splat-cooled U-T alloys. The two additional peaks also cannot be associated with the binary U-Pt phase (U-Pt with monoclinic structure of the PdBi type, mP16, space group P $2_{1}$ ), which should have the two strongest peaks at $33^{\circ}$ and $41^{\circ}$ [22]. The XRD pattern of U-15 at.\% Pt revealed four characteristic peaks of the $\gamma$ type structure $[\gamma(110), \gamma(200), \gamma(211)$ and $\gamma(220)$, respectively, at $36.8^{\circ}, 53.0^{\circ}, 65.3^{\circ}$ and $78.2^{\circ}$ ], indicating a stabilization of the cubic $\gamma-\mathrm{U}$ phase. However, unlike U-15 at.\% Mo with very narrow $\gamma$-peaks indicating the reflections of the fundamental cubic A2 structure, there is a certain broadening for all the $\gamma$-peaks in U-15 at.\% Pt, similar to that observed in U-13 at.\% Mo splat. It is interesting to compare our findings with respective binary phase diagrams. The maximum reported solubility in $\gamma-\mathrm{U}$ of $\mathrm{Pt}$ or $\mathrm{Pd}$ does not exceed 5 at.\% [7, 22-24]. Our results reveal that using the splat cooling we not only retain the $b c c$ phase to low temperatures, but also extend its occurrence for much higher concentrations of alloying Pt/Pd metals.
The normalized XRD patterns of the splat-cooled U-Nb alloys in the as-formed state are shown in Fig. 3b. In general, the increase in the $\mathrm{Nb}$ concentration leads to the suppression of $\alpha$-U reflections and the development of $\gamma-\mathrm{U}$ reflections. It causes the overlap of low-index peaks around $36^{\circ}$, and then the combined peak becomes narrower for 10 at.\% $\mathrm{Nb}$. For the U-15 at.\% $\mathrm{Nb}$ alloy, the splitting of the $\gamma$-reflections into doublets was observed for all four prominent $\gamma$-peaks. For instance, the $\gamma$ (110) reflection of $\mathrm{U}-15$ at. $\% \mathrm{Nb}$ splits into doublet located around $36.3^{\circ}$ $\left[\gamma^{0}(101)\right]$ and $37.0^{\circ}\left[\gamma^{0}(110)\right]$. The situation is similar to that of doping with 11-12 at.\% Mo which stabilizes the $\gamma^{0}$ $\mathrm{U}$ phase (the body-centered tetragonal structure with the $c$ l $a$ ratio of about $0.98-0.99)$. In general, our results show a similarity between the U-Nb and U-Mo systems. It is in a good agreement with an earlier observation revealing that the mode of formation of $\alpha-, \alpha^{\prime}$ - and $\gamma^{0}$-phase in the $\mathrm{U}-\mathrm{Nb}$ system is very similar to that observed in the U-Mo system. Moreover, we expect that using ultrafast cooling could reduce the necessary $\mathrm{Nb}$ concentration. Indeed, it turned out that the $\gamma^{0}-\mathrm{U}$ phase is found to be stabilized by 15 at.\% $\mathrm{Nb}$ doping, i.e., lower than the minimal content for stabilization of such a phase in water-quenched (16.8 at.\% $\mathrm{Nb}$ ) 

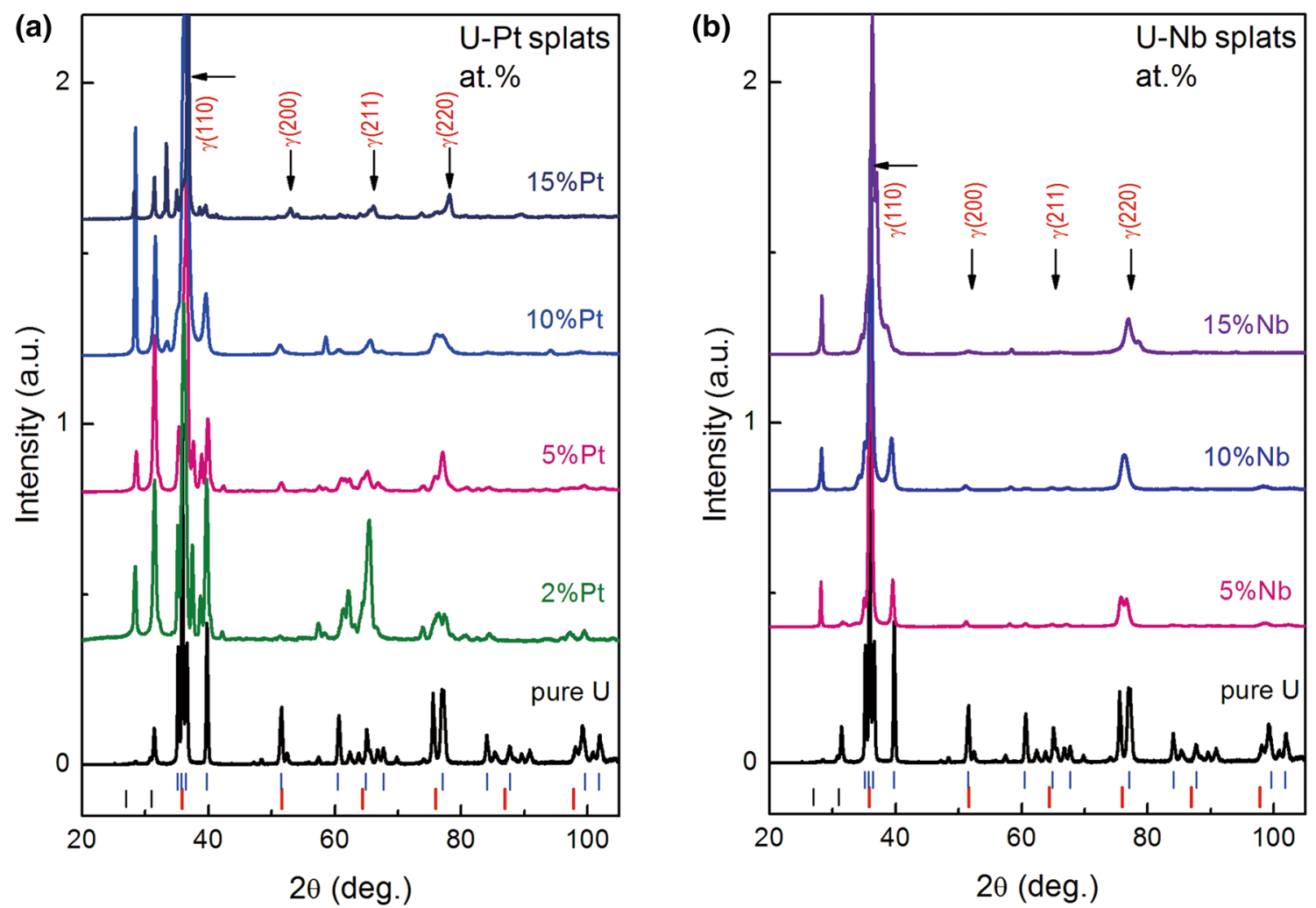

Fig. 3 XRD patterns of the as-formed splat-cooled U-Pt alloys a and U-Nb alloys $\mathbf{b}$. Each curve was normalized to the maximal intensity of the most intense peak at $2 \theta=36^{\circ}-37^{\circ}$ and then shifted upwards with respect to that of pure $\mathrm{U}$ splat. The color vertical ticks indicate the main XRD patterns of orthorhombic (blue) and cubic (red) structures and of the surface impurities (black). The four main $\gamma$-reflections are also indicated

[25] or in argon-quenched ones (16.2 at.\% Nb) [26]. Using a combined arc melting, hot rolling, annealing and water quenching, the $\gamma$-U phase was stabilized in $\mathrm{U}-7 \mathrm{wt} \% \mathrm{Nb}$ ( $\sim \mathrm{U}-15$ at.\% $\mathrm{Nb}$ ) alloy [27].

In the case of the $\mathrm{U}-\mathrm{Zr}$ system, there is a similar situation to for $\mathrm{U}-\mathrm{Nb}$, i.e., the complete miscibility of the hightemperature $b c c$ phase. The normalized XRD patterns of the splat-cooled $\mathrm{U}-\mathrm{Zr}$ alloys in the as-formed state are shown in Fig. 4a. The results illustrate the phase transformation from the $\alpha$-phase to $\gamma$ with increasing $\mathrm{Zr}$ concentration. Unlike other $T$ doping, the $\alpha(110)$ and $\alpha(111)$ peaks still persist for $\mathrm{U}-11$ at. $\% \mathrm{Zr}$ and $\mathrm{U}-15$ at.\% $\mathrm{Zr}$, but become very broad for $\mathrm{U}-20$ at. $\% \mathrm{Zr}$ and then vanish for $\mathrm{U}-30$ at. $\%$ $\mathrm{Zr}$, as shown in more detail in an enlarged scale in Fig. 4b. Existing reports indicate that the single-phase $\gamma$-alloys were obtained for $\mathrm{Zr}$ concentrations between 25 and 80 at.\% [28]. In our case, the $\gamma$-U reflections increase visibly with increasing $\mathrm{Zr}$ concentration and become dominant for all splat samples with $\mathrm{Zr}$ concentrations $\geq 11$ at. $\%$. We notice here that U-7wt \% Zr ( U-15 at.\% Zr) alloy prepared by a combined arc melting, hot rolling, annealing and water quenching has revealed still a mixed $\alpha+\gamma-\mathrm{U}$ phase [27]. In our case, the single $\gamma-\mathrm{U}$ phase can be considered only for $\mathrm{U}-30$ at.\% $\mathrm{Zr}$ splat. Moreover, most of the $\gamma$-reflections [including the main peak $\gamma(111)$ at $35.9^{\circ}$ ] are broadened.
At low $\mathrm{Zr}$ concentrations, the broadening can be seen in the overlap of the $\gamma$-peaks with the $\alpha$-peaks. But at high $\mathrm{Zr}$ concentrations, the contribution from $\alpha$-peaks was minimized. Thus, the main reason for the peak broadening should be the additional disorder (microstrain) by randomly distributed $\mathrm{Zr}$ atoms (less probable due to almost identical atomic size) and/or limited grain size due to splat cooling. This suggestion is confirmed by the fact that the peak broadening increases with $\mathrm{Zr}$ concentration. Similar to other splats, UC (111) and $\mathrm{UO}_{2}$ (111) impurity peaks were observed in the low-angle part of the XRD patterns. These impurities are segregated at the surface of the splats and can be easily removed by the sample scraping; i.e., these impurity peaks are largely reduced after scraping. Narrow peaks of $\mathrm{ZrC}$ were observed for $\mathrm{U}-\mathrm{Zr}$ samples with the most intense reflections $\mathrm{ZrC}$ (111) and $\mathrm{ZrC}$ (200) at $33.4^{\circ}$ and $38.7^{\circ}$, respectively. Traces of carbon are ubiquitous in uranium metal, and it seems that it couples preferentially with $\mathrm{Zr}$ when it enters the alloy, and moreover it has a high surface segregation tendency. We also notice that no sign of the phase $\mathrm{UZr}_{2}$ ( $\delta$-phase) was found in XRD patterns even if for the samples with high $\mathrm{Zr}$ content (20 and 30 at. $\% \mathrm{Zr}$ ).

For more details, we show in Fig. 5 the comparison of the most intense reflection in XRD patterns at around $37^{\circ}$ 

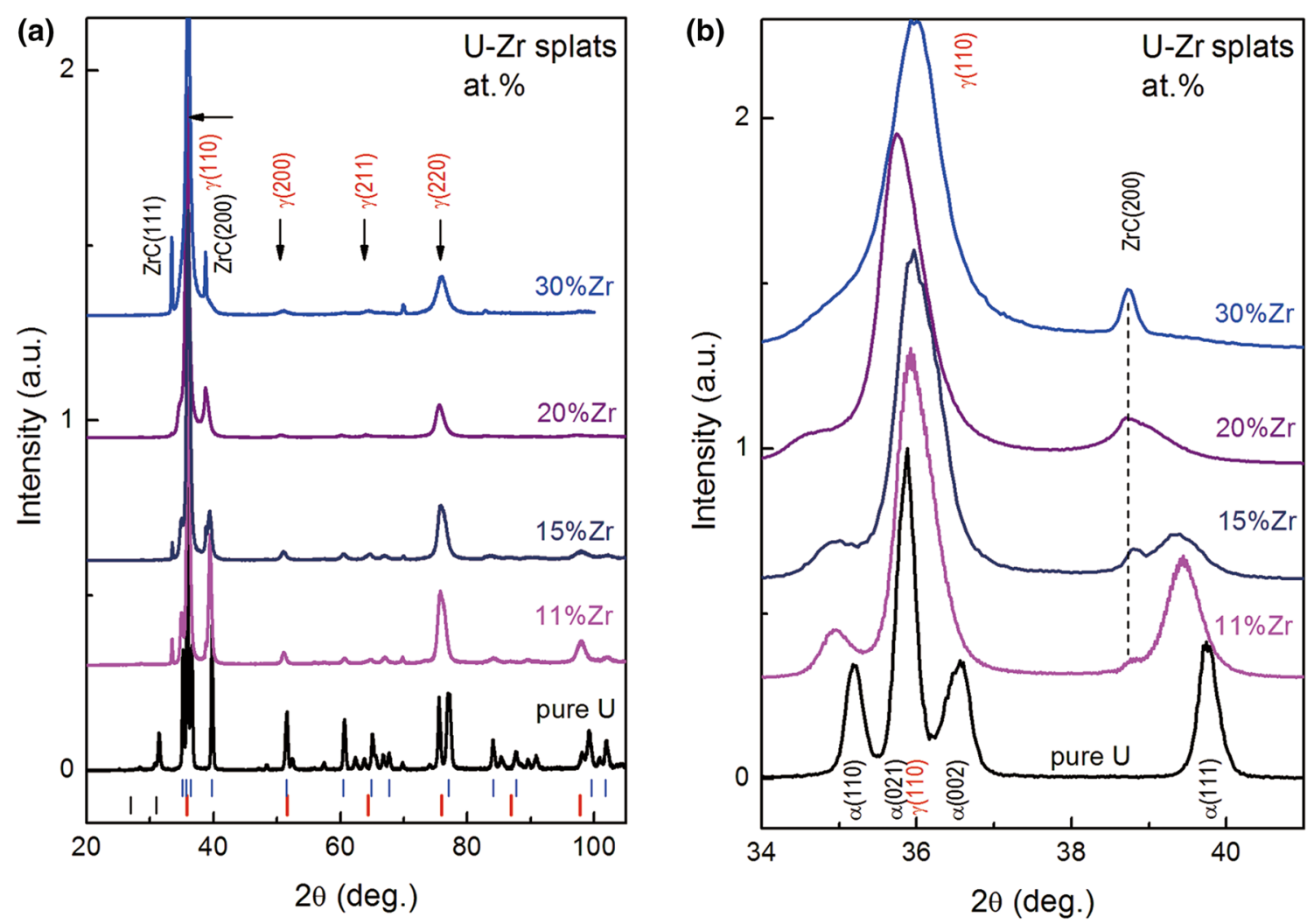

Fig. 4 XRD patterns of the as-formed splat-cooled U-Zr alloys shown as normalized ones a. The same notations of the color vertical ticks are used as those in Fig. 3. The enlarged low-angle XRD patterns b. For U-Zr splats, apart from surface impurities $\mathrm{UO}_{2}$ and $\mathrm{UC}_{\text {, the }}$ peaks of spurious $\mathrm{ZrC}$ are observed

of as-formed splat-cooled U-15 at.\% $T$ splats, i.e., with the same amount of 15 at.\% $T$ alloying. A single sharp $\gamma$-peak and no trace of any $\alpha$-peaks were observed only for U-15 at.\% Mo consisting of a pure single $b c c$ structure $(\gamma-$ $\mathrm{U}$ phase). The $b c c$ structure could be also stabilized in U-15 at.\% Pt (combined with ultrafast cooling), but a small trace of $\alpha-\mathrm{U}$ phase is still present. Alloying with 15 at. $\%$ $\mathrm{Nb}$ could stabilize the $\gamma^{0}-\mathrm{U}$ phase, but with a larger broadening of $\gamma^{0}$-peaks, in comparison with those for U-12 at.\% Mo splat. We also add to the figure the data for $\mathrm{U}-30$ at.\% $\mathrm{Zr}$ exhibiting the $\gamma-\mathrm{U}$ phase. The $\gamma(110)$ peak is relocated to lower angles reflecting a lattice expansion. We have also investigated the crystal structure of splats with combined 12 at.\% Mo plus 3 at.\% $\mathrm{Zr}$ alloying, i.e., replacing 3 at.\% Mo in U-15 at.\% Mo by 3 at.\% Zr [U15 at.\% (Mo $+\mathrm{Zr})]$. A doublet splitting was observed for all $\gamma$-peaks. The peaks are quite narrow, similar to those of U-12 at.\% Mo. Besides, the most intense doublet assigned to $\gamma^{0}(101)$ and $\gamma^{0}(110)$ reflections (at $36.8^{\circ}$ and $37.0^{\circ}$, respectively) of $\mathrm{U}-15$ at.\% (Mo $+\mathrm{Zr}$ ) is more symmetric and with roughly equal intensity. In other words, exchanging 3 at.\% Mo by 3 at.\% $\mathrm{Zr}$ doping implies a change from pure $b c c$ structure $[\gamma-\mathrm{U}$ phase (in $\mathrm{U}-15$ at. $\%$ Mo)] into a body-centered tetragonal structure $\left(\gamma^{0}-\mathrm{U}\right.$ phase).
The lattice parameters estimated for the $\gamma$-U phase alloys are given in Table 1 . The atomic radius of $\mathrm{Nb}(1.45 \AA), \mathrm{Pd}$ $(1.40 \AA)$ and Pt $(1.35 \AA)$ was equal or close to that of Mo $(1.45 \AA)$, all of which are lower than the nominal atomic radius of $\mathrm{U}(1.56 \AA)$, while the $\mathrm{Zr}$ atomic radius $(1.60 \AA)$ is larger [29]. We may consider that replacing some Mo by $\mathrm{Zr}$ atoms would induce a tetragonal distortion of the cubic lattice and/or some shifts of the atoms from their ideal A2 sites. We notice here also that the $\gamma(110)$ reflection in $\mathrm{U}-15$ at. $\% \mathrm{Nb}$ and in $\mathrm{U}-15$ at.\% (Mo $+\mathrm{Zr}$ ) locates at $37.0^{\circ}$, i.e., at the angle similar to that of the pure cubic $\mathrm{U}-15$ at.\% Mo alloy, indicating that the $a$ parameter of the tetragonal structure in these alloys is similar to that of the $b c c$ structure of U-15 at.\% Mo. The earlier observation indicated that for the U- $T$ alloys, the $a$ parameter is not affected visibly by alloying, while the $b$ and $c$ parameters exhibit some variations. Besides, the atomic radius of $\mathrm{Nb}$ is similar to that of Mo. Thus, doping with 15 at.\% $\mathrm{Nb}$ or exchanging a part of Mo by $\mathrm{Zr}$ (3 at.\%) should not lead to any visible change of the unit cell. But it is not clear why $\mathrm{Nb}$ doping implies much broader doublets than those with Mo doping, despite that $\mathrm{Nb}$ is an immediate neighbor of Mo. The $\gamma$ (110) reflection in $\mathrm{U}-15$ at.\% $\mathrm{Pt}$ is located at a lower angle $\left(36.8^{\circ}\right)$. It indicates that, although the 15 at. $\%$ Pt doping leads to a stabilization of the bcc structure, 
smaller Pt atoms (in comparison with Mo atoms) can lead also to a visible microstrain [broader $\gamma$ (110) reflection], although the lattice parameter of the two alloys is similar. Doping with $\mathrm{Zr}$, i.e., with larger atoms, certainly leads to a large expansion of the lattice. The relative increase in the lattice $(\Delta a)$ is estimated to be about $3 \%$ for 30 at. $\% \mathrm{Zr}$ alloying.

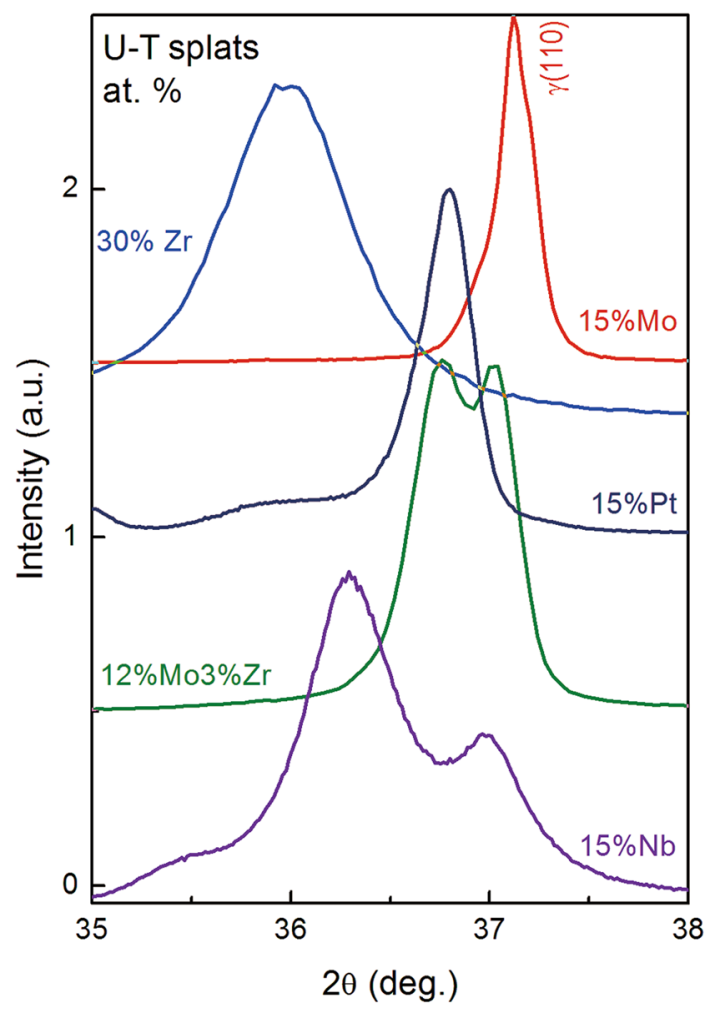

Fig. 5 Comparison of the most intense reflection in XRD patterns of $\mathrm{U}-T$ splats with 15 at. $\% T$ alloying $[T=\mathrm{Mo},(\mathrm{Mo}+\mathrm{Zr}), \mathrm{Pt}, \mathrm{Nb}]$ in the as-formed state. Sharp $\gamma$-phase peaks and no trace of $\alpha$-phase peaks were observed for only U-15 at.\% Mo splat

Table 1 Summary of low-temperature properties of U-T splat alloys: resistivity values at $300 \mathrm{~K}$ and at $4 \mathrm{~K}\left(\rho_{300 \mathrm{~K}}, \rho_{4 \mathrm{~K}}\right)$, superconducting transition temperatures $\left(T_{\mathrm{c}}\right)$ determined from the $\rho(T)$ jump and/or from the specific heat $C(T)$, the width of the superconducting

\subsection{Superconductivity in the U-T Splats Stabilized in the Cubic $\gamma$-U Phase}

\subsubsection{Electrical Resistivity and Superconductivity in $U-M o$ Splats}

For a brief summary of the change of the temperature coefficient in splat-cooled $\mathrm{U}-T$ alloys with increasing $T$ content in the normal state in the temperature range 3-300 K, we show in Fig. 6 the temperature dependence of the (normalized) electrical resistivity of U-Mo splats (the data were reported separately earlier $[18,30,31])$. We concentrate on analyzing the two limit cases (Fig. 6a), which reveal a striking difference in the pure $U$ splat (consisting of $\alpha-\mathrm{U}$ phase) and the $\mathrm{U}-15$ at.\% Mo splat (consisting of the $\gamma-\mathrm{U}$ phase only). The pure $\mathrm{U}$ splat exhibits a quadratic temperature dependence below $50 \mathrm{~K}$ and then an almost linear dependence up to $300 \mathrm{~K}$, i.e., with a positive temperature coefficient $(\mathrm{d} \rho / \mathrm{d} T>0)$. Unlike such a common metallic behavior, for U-15 at.\% Mo, the resistivity weakly decreases with increasing temperature in the normal state in the whole temperature range, i.e., with a negative temperature coefficient $(\mathrm{d} \rho / \mathrm{d} T<0)$. The temperature dependence of the resistivity of other U-Mo splats lies between such the two limits. In other words, increasing the Mo content leads to a change from metallic $(\mathrm{d} \rho /$ $\mathrm{d} T>0)$ to nonmetallic $T$ dependence $(\mathrm{d} \rho / \mathrm{d} T<0)$. Besides, while U-Mo alloys consisted of both $\alpha$ - and $\gamma$ phases (with $<10 \%$ Mo doping) have still positive $\mathrm{d} \rho / \mathrm{d} T$, all $\gamma$-phase U-Mo alloys (with $\geq 11 \%$ Mo doping) have the negative $\mathrm{d} \rho / \mathrm{d} T$. We suggest that a large disorder effect plays an important role in the splat-cooled alloys, similar to a strong disorder observed, e.g., in some (superconducting) amorphous system [32], Heusler alloys [33] and disordered heavy-fermion compound $\mathrm{URh}_{2} \mathrm{Ge}_{2}$ [34]. The reason for the negative slope can be seen in the weak localization, i.e., a quantum interference effect (e.g., the anomalous

transition in the resistivity $\left(\Delta T_{\rho}\right)$, the Sommerfeld coefficient of electronic specific heat $\left(\gamma_{\mathrm{e}}\right)$ and Debye temperature $\left(\Theta_{\mathrm{D}}\right)$. The structure and lattice parameters $(a, c)$ are also given

\begin{tabular}{|c|c|c|c|c|c|c|c|c|c|}
\hline$T$ content (at. $\%$ ) & Type & $a, c(\AA)$ & $\rho_{300 \mathrm{~K}}(\mu \Omega \mathrm{cm})$ & $\rho_{4 \mathrm{~K}}(\mu \Omega \mathrm{cm})$ & $T_{\mathrm{c}}(\mathrm{K})[\rho(T)]$ & $\Delta T_{\rho}(\mathrm{K})$ & $T_{\mathrm{c}}(\mathrm{K})[C(T)]$ & $\gamma_{\mathrm{e}}\left[\mathrm{mJ} /\left(\mathrm{K}^{2} \mathrm{~mol}\right)\right]$ & $\Theta_{\mathrm{D}}(\mathrm{K})$ \\
\hline Pure U & $\alpha$ & & 53 & 14 & 1.24 & 0.20 & 0.65 & 11.0 & 179 \\
\hline $15 \% \mathrm{Mo}$ & $\gamma$ & 3.441 & 89 & 95 & 2.11 & 0.02 & 2.11 & 16.0 & 139 \\
\hline $15 \% \mathrm{Pt}$ & $\gamma$ & 3.469 & 164 & 166 & $0.95 / 0.61$ & $0.08 / 0.04$ & 0.75 & 19.5 & 145 \\
\hline $15 \% \mathrm{Nb}$ & $\gamma^{0}$ & $\begin{array}{l}3.435(a) \\
3.565(c)\end{array}$ & 83 & 86 & 1.90 & 0.15 & 1.90 & 13.7 & 153 \\
\hline $15 \%(\mathrm{Mo}+\mathrm{Zr})$ & $\gamma^{0}$ & $\begin{array}{l}3.431(a) \\
3.482(c)\end{array}$ & & & & & & & \\
\hline $30 \% \mathrm{Zr}$ & $\gamma$ & 3.543 & 75 & 73 & 0.81 & 0.08 & 0.60 & 11.8 & 165 \\
\hline
\end{tabular}



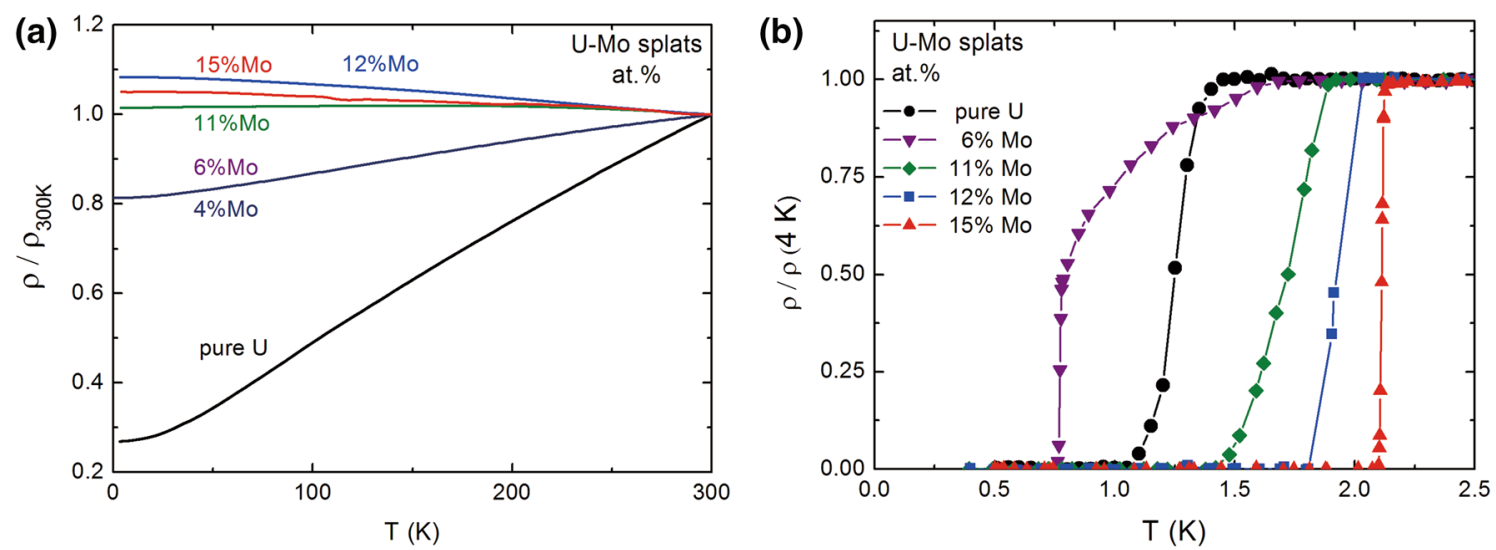

Fig. 6 Temperature dependence of electrical resistivity (in zero field) of U-Mo splats in the normal state a and around the superconducting transition temperature b. For an easier comparison, the curves were normalized to respective resistivity values at $T=300 \mathrm{~K}$ and at $T=4 \mathrm{~K}$. All U-Mo splats with the $\gamma$-U phase ( $\geq 11$ at.\% Mo doping) have a negative temperature coefficient $(\mathrm{d} \rho / \mathrm{d} T<0)$. The pure single $\gamma$-U phase $\mathrm{U}-15 \%$ Mo splat has a highest critical temperature $\left(T_{\mathrm{c}}=2.11 \mathrm{~K}\right)$ and sharpest resistivity drop

dispersion of the conduction electrons) occurring in strongly disordered systems [35]. In our case, there is certainly still some extra contribution to the disorder produced by ultrafast cooling, affecting the grain size, but the fact that $\mathrm{d} \rho / \mathrm{d} T<0$ found in all U-Mo alloys with $b c c$ structure reveals a dominant role of strong conduction electron scattering on randomly distributed $\mathrm{U}$ and Mo ions.

All investigated U-Mo splats become superconducting at low temperatures. The superconducting transitions revealed by abrupt resistivity drops in zero magnetic field are shown in Fig. 6b. We focus firstly on the data for the two limits: the pure $\mathrm{U}$ splat and the U-15 at.\% Mo splat. The transition is manifest as a single drop at $T_{\mathrm{c}}=1.24 \mathrm{~K}$ and $2.11 \mathrm{~K}$, respectively $[18,30]$. We remind here a very small width of the transition $\Delta T_{\rho}=0.02 \mathrm{~K}$ observed for U-15 at. $\% \mathrm{Mo}$, while a wider transition $\Delta T_{\rho}=0.2 \mathrm{~K}$ was found for pure $\mathrm{U}$ splat. However, unlike a $\lambda$-type anomaly for $\mathrm{U}-15$ at.\% Mo, the superconducting transition in pure $\mathrm{U}$ splat was revealed only as a small feature around $0.65 \mathrm{~K}$ in the specific heat [18]. It is an evidence against the bulk nature of superconductivity. It can be understood only a small fraction of the sample becomes superconducting, and as it has to form a 3D network, it must be related to the grain boundaries. For other $\gamma$-U alloys, such as U-11 at.\% Mo and U-12 at.\% Mo, the superconducting transition also appears as a single resistivity drop, although broader than that in U-15 at.\% Mo. We pay particularly attention to the superconducting transition in the U-6 at.\% Mo splat [21]; i.e., the intermediate range of Mo doping consisted of both $\alpha$ - and $\gamma$-U phases. The phase coexistence is reflected by a flat but still a metallic type overall temperature dependence $(\mathrm{d} \rho / \mathrm{d} T>0)$. In the low- $T$ range, the resistivity starts to decrease rapidly below $1.6 \mathrm{~K}$, which has to be interpreted as emergence of a small amount of superconducting phase. This decrease ends in an abrupt drop into the zero resistance state at $T_{\mathrm{c}}=0.78 \mathrm{~K}$. The obtained results show that there are two different superconducting phases in the U-6 at.\% Mo splat, (we have to assume the coexisting $\alpha$ and $\gamma$-U phase), each of which exhibits its own superconductivity. We speculate that the superconductivity of the $\alpha$ phase is restricted to surface of the grains, but not forming the complete 3D network as the phase is not contiguous, which succeeds to reduce the total resistivity of the composite structure, but has a very little impact on the specific heat. The lower $T_{\mathrm{c}}$ may be associated with the $\gamma-\mathrm{U}$ phase, as it revealed by a sizeable anomaly in the specific heat [21]. We cannot, however, exclude that the higher $T_{\mathrm{c}}$ belongs to the $\gamma-\mathrm{U}$ phase, if that would represent only a very small fraction, assuming that the $\alpha-\mathrm{U}$ phase with Mo can have a bulk superconductivity in which Mo prevents the CDW formation.

\subsubsection{Superconductivity in $U-T$ Splats $(T=P t, P d, N b$, $\mathrm{Zr}$ ) with $\gamma$-U Phase}

As next step, we focus on a comparison of the resistivity behavior of all splat-cooled $\mathrm{U}-T$ alloys $(T=\mathrm{Pt}, \mathrm{Pd}, \mathrm{Nb}$, $\mathrm{Zr}$ ) crystallized in the $\gamma-\mathrm{U}$ structure. The temperature dependence of the resistivity of these alloys in zero field and in the temperature range of $3-300 \mathrm{~K}$ is shown in Fig. 7a. The resistivity values at $300 \mathrm{~K}$ and $4 \mathrm{~K}$ are given in Table 1. The $\rho(T)$ curves of $\mathrm{U}-15$ at. $\% \mathrm{Mo}$ and $\mathrm{U}-15$ at. $\% \mathrm{Nb}$ splat are quite similar. Besides, the residual resistivity $\rho_{0}\left(\rho_{4} \mathrm{~K}\right)$ and the resistivity at room temperature $\left(\rho_{300 \mathrm{~K}}\right)$ are also similar (see Table 1$)$. For the U-15 at.\% Pt splat, although the resistivity values are twice higher, the relative change of the resistivity in U-15 at.\% Pt [the $\rho /$ $\rho_{300 \mathrm{~K}}(T)$ curve] is very similar to that of U-15 at.\% Mo (as well as U-15 at.\% Nb). Namely, from room temperature down to temperature just above the superconducting 
transition, the electrical resistivity exhibits a negative temperature coefficient $(\mathrm{d} \rho / \mathrm{d} T<0)$. For $\mathrm{U}-30$ at. $\% \mathrm{Zr}$ revealing the $\gamma$-U phase, the negative slope does not develop yet. Instead, we found a very small (close to zero) but still positive slope of the temperature dependence in this splat. We like to mention that a negative temperature coefficient $(\mathrm{d} \rho / \mathrm{d} T<0)$ was indeed reported for $\mathrm{U}-\mathrm{Zr}$ system, but for sample with 70 at.\% Zr [36]. We suggest that the negative slope can be also observed for $\mathrm{U}-\mathrm{Zr}$ splats, but certainly for higher $\mathrm{Zr}$ concentrations than 30 at.\%. The low-temperature $\rho(T)$ dependence of U-15 at $\% T(T=\mathrm{Mo}, \mathrm{Nd}, \mathrm{Pt})$ splats measured in zero field is shown in Fig. 7b. For comparison, we also show in the same figure the data for pure $\mathrm{U}$ splat as well as those for $\mathrm{U}-30$ at. $\% \mathrm{Zr}$ splat (consisted of $\gamma-\mathrm{U}$ phase). In all cases, a very sharp resistivity drop was observed at $T_{\mathrm{c}}$. The sharpest superconducting transition for all investigated $\gamma-\mathrm{U}$ phase alloys was observed in U-15 at. $\%$ Mo $\left(\Delta T_{\rho}=0.02 \mathrm{~K}\right)$. $\mathrm{U}-15$ at. $\% \mathrm{Nb}$ becomes superconducting at the superconducting transition temperature $T_{\mathrm{c}}=1.90 \mathrm{~K}$, revealing as a single sharp drop at $T_{\mathrm{c}}$ with a transition width $\Delta T_{\rho}=0.15 \mathrm{~K}$. It is noted that $\mathrm{U}-15$ at. $\% \mathrm{Nb}$ has the $\gamma^{0}-\mathrm{U}$ structure. Except of a small difference in the $T_{\mathrm{c}}$ values, the resistivity drop in $\mathrm{U}-15$ at.\% $\mathrm{Nb}$ is very similar to that of other splat alloys consisting of $\gamma^{0}-\mathrm{U}$ structure (with $11-12$ at.\% Mo). The superconductivity in $\mathrm{U}-15$ at.\% Pt was characterized by a sharp drop at $T_{\mathrm{c}}=0.61 \mathrm{~K}$ with a very small transition width $\Delta T_{\rho}=0.04 \mathrm{~K}$. Despite of a similarity in the crystal structure $(\gamma-\mathrm{U})$ and lattice parameter between U-15 at.\% Mo and U-15 at.\% Pt (resulted from alloying with elements with a similar atomic radius), U-15 at.\% Pt becomes superconducting at much lower temperature. Besides, an extra feature was observed at $0.95 \mathrm{~K}$ with a transition width $\Delta T_{\rho}=0.08 \mathrm{~K}$. The origin of this extra drop is still unknown. More detailed investigations of the superconducting phase transition in
$\mathrm{U}-15$ at.\% Pt are in progress. We notice here, even if for the splat-cooled U-5 at.\% Pt alloy consisting of a mixed $\alpha$ $\mathrm{U}$ and $\gamma-\mathrm{U}$ phase, the superconducting phase transition is revealed by only a single resistivity drop [21]. Our study shows that double drops are also observed in the U-15 at.\% Pt bulk alloy (the precursor of the splat-cooled sample). U-30 at.\% Zr exhibits a superconducting transition which is revealed by a single drop at $T_{\mathrm{c}}=0.81 \mathrm{~K}$ with a transition width $\Delta T_{\rho}=0.08 \mathrm{~K}$.

Applying external magnetic fields, the superconducting transitions shift toward lower temperatures, as expected. The estimated values for critical magnetic fields at zero temperature $\left(\mu_{0} H_{\mathrm{c}}\right)$ and the critical slopes at $T_{\mathrm{c}}$ of the $H_{\mathrm{c} 2}$ versus $T$ curves $\left[-\mu_{0}\left(\mathrm{~d} H_{\mathrm{c} 2} / \mathrm{d} T\right)_{T \mathrm{c}}\right]$ for selected U-Mo splats are reported earlier $[18,19]$. In Table 1 , we list only the values for pure $\mathrm{U}$ and $\mathrm{U}-15$ at.\% Mo splat, for a comparison with other $T$-doping splats.

Applying external magnetic fields, the superconducting transitions shift toward lower temperatures, as expected. The estimated values for $\left(\mu_{0} H_{\mathrm{c}}\right)$ and $\left[-\mu_{0}\left(\mathrm{~d} H_{\mathrm{c} 2} / \mathrm{d} T\right)_{T \mathrm{c}}\right]$ are in the range of 2-7 T and 2-4 T/K (see Table 1), respectively. These values are close to that found for the strongly interacting Fermi liquid superconductor $\mathrm{U}_{6} \mathrm{Fe}\left[-\mu_{0}\left(\mathrm{~d} H_{\mathrm{c} 2} /\right.\right.$ $\mathrm{d} T)_{T \mathrm{c}}=3.42 \mathrm{~T} / \mathrm{K}$ ] [37] as well as within the limit for the extreme high-field A15 and Chevrel-phase superconductors $2 \mathrm{~T} / \mathrm{K} \leq\left[-\mu_{0}\left(\mathrm{~d} H_{\mathrm{c} 2} / \mathrm{d} T\right)_{T \mathrm{c}} \leq 8 \mathrm{~T} / \mathrm{K}\right]$ [38]. One difference is that for those splat-cooled $\gamma-\mathrm{U}$ alloys, the $T_{\mathrm{c}}$ values are lower than $2.2 \mathrm{~K}$, while Chevrel-phase superconductors have much higher $T_{\mathrm{c}}(>10 \mathrm{~K})$.

\subsubsection{Specific-Heat Anomaly at the Superconducting Phase Transition of U-T Splats}

The temperature dependence of specific heat, $C_{\mathrm{p}}(T)$, has been studied for selected splats over the whole temperature range, including both the low- $T$ and high- $T$ parts for
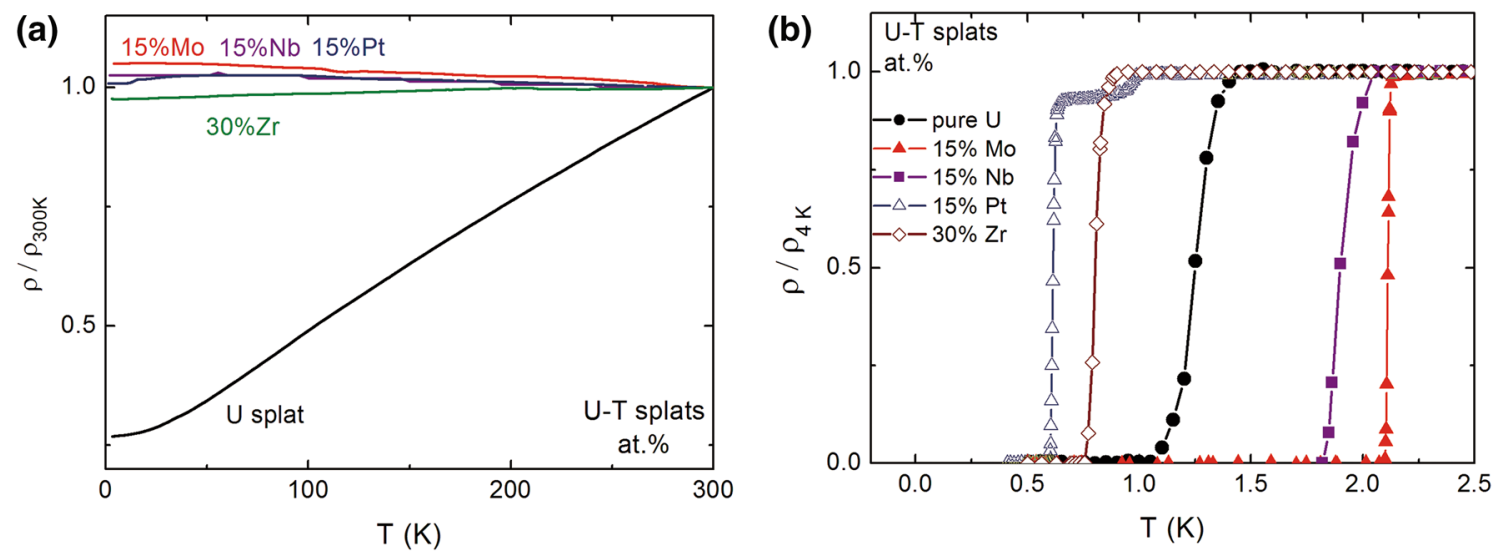

Fig. 7 Temperature dependence of the normalized electrical resistivity in zero field of splat-cooled $\mathrm{U}-T$ splats having the $\gamma$-U phase compared with that of pure uranium splat a. All the alloys with 15 at.\% $T$ doping $(T=\mathrm{Mo}, \mathrm{Nb}, \mathrm{Pt})$ have a negative $\mathrm{d} \rho / \mathrm{d} T$, except for $\mathrm{U}-30 \mathrm{at} . \% \mathrm{Zr}$ having a positive one. Superconducting phase transitions indicate by the abrupt resistivity drops in the range of $0.61-2.11 \mathrm{~K} \mathrm{~b}$ 
characterizing the superconducting behavior as well as the electronic and phonon contribution. The Sommerfeld coefficient of electronic specific heat $\left(\gamma_{e}\right)$ and the Debye temperature $\left(\Theta_{\mathrm{D}}\right)$ can be estimated from the $C_{\mathrm{p}} / T$ versus $T^{2}$ plot in the normal state $\left[C_{\mathrm{p}} / T=\gamma_{e}+\beta T^{2} ; \beta=1944 /\right.$ $\left(\Theta_{\mathrm{D}}\right)^{3}$ for $C_{\mathrm{p}}$ in $\left.\mathrm{J} /(\mathrm{mol} \mathrm{K})\right]$. The estimated values are given in Table 1. A clear evidence of an increase in density of states at the Fermi level for $\gamma-\mathrm{U}$ is observed only for $\mathrm{U}-15$ at.\% Mo, as shown by an enhancement of the $\gamma_{e}$ value by Mo doping $\left[\gamma_{e}=16-18.8 \mathrm{~mJ} /\left(\mathrm{K}^{2} \mathrm{~mol}\right) \mathrm{U}\right.$ for $\mathrm{U}-15$ at.\% Mo, in comparison with that for pure U $\left.\gamma_{e}=11 \mathrm{~mJ} /\left(\mathrm{K}^{2} \mathrm{~mol}\right) \mathrm{U}\right]$. It is ascribed to the increasing atomic volume and higher U-U spacing. The enhancements of the $\gamma_{e}$ value and the softening of the lattice (revealed by reduction in Debye temperature) are found to be smaller for $\mathrm{Nb}$ and $\mathrm{Zr}$ alloying.

The temperature dependence of the specific heat and its field variations have been performed down to $0.3 \mathrm{~K}$ for selected splat-cooled $\mathrm{U}-T$ alloys. The jump in the specific heat at $T_{\mathrm{c}}$ within the BCS theory in the weak coupling approximation is:

$\Delta C=1.43 \gamma_{\mathrm{e}} T_{\mathrm{c}}$.

We estimated the height of the experimentally observed specific-heat jump $(\Delta C)$ and then compared to the estimated BCS values by using the $\gamma_{\mathrm{e}}$ and $T_{\mathrm{c}}$ values determined from our experiments.

In Fig. 8, we show the $C-T$ curves in zero field for the two limits: the pure $\mathrm{U}$ and $\mathrm{U}-15$ at.\% Mo splat. Only a very small feature related to the superconducting transition was revealed at $0.65 \mathrm{~K}$ in the specific heat for the pure $\mathrm{U}$ splat. The results suggest that only a small fraction of the sample is really superconducting. For U-15 at.\% Mo splat (consisted of single $\gamma$-U phase with ideal $b c c$ A2 structure), a pronounced $\lambda$-type specific-heat anomaly was observed. The height of the experimentally observed specific-heat jump $(\Delta C)$ is in a good agreement with that estimated from BCS theory (see Fig. 5 Ref. [30]). Such a robust BCS-type superconductivity culminating for U-15 at.\% Mo revealed that it might be in analogy with that of $\mathrm{U}_{6} \mathrm{Fe}$ [37]. For other U-Mo splats with lower Mo contents ( $<15$ at.\%), a broader peak with a smaller (but nonnegligible) specific-heat jump was observed close to the superconducting transition temperature $T_{\mathrm{c}}$ defined from the resistivity measurements. The experimentally estimated jump for instance for U-6 at.\% Mo splat amounts to only about $55 \%$ of the BCS value [21]. The specific heat of other $\mathrm{U}-T$ splats consisted of $\gamma-\mathrm{U}$ phase measured down to $0.3 \mathrm{~K}$ in zero magnetic field was shown also in Fig. 8. Only a weak and broad bump with a small height was observed in $C(T)$ curve of $\mathrm{U}-15$ at. $\% \mathrm{Nb}$. Although the crystal structure, the resistivity jump and $T_{\mathrm{c}}$ value of this splat are similar to that of U-12 at.\% Mo splat, much a larger peak was observed for U-12 at.\% Mo in the

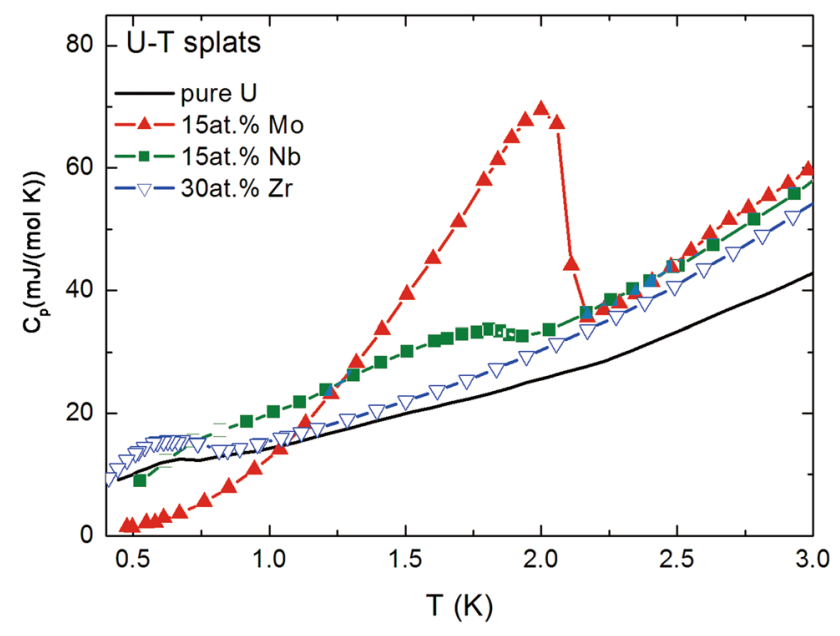

Fig. 8 Specific-heat anomalies related the superconducting phase transition for selected $U-T$ splats. A pronounced $\lambda$-type specific-heat anomaly was observed only for U-15 at.\% Mo splat consisting of single $\gamma$-U phase with ideal $b c c$ A2 structure

$C(T)$ curve. The specific heat peak related to the superconducting transition in $\mathrm{U}-30$ at. $\% \mathrm{Zr}$ splat is visible at $T_{\mathrm{c}}$ determined from the resistivity jump, revealing that the superconductivity in this splat is a real bulk effect.

We notice here also that in the context of the stability of the $b c c \mathrm{U}-T$ alloys it is interesting to mention their high resistance to hydrogen absorption. While $\alpha-\mathrm{U}$ easily absorbs hydrogen even at room temperature and few mbar of $\mathrm{H}_{2}$ forming $\mathrm{UH}_{3}$, the $\mathrm{U}-T$ alloys with cubic $\gamma-\mathrm{U}$ phase do not absorb hydrogen in the hydrogen atmosphere up to hydrogen pressure of $2.5 \times 10^{5} \mathrm{~Pa}(2.5 \mathrm{bar})$; i.e., they are highly resistance against hydrogen absorption. We have demonstrated that elevated pressures $p\left(\mathrm{H}_{2}\right)>2.5$ bar are needed to achieve hydrides of equivalent stoichiometry for $\mathrm{U}-\mathrm{Mo}$ and $\mathrm{U}-\mathrm{Zr}$ alloys. The lattice expansion due to hydrogen absorption leads to the disappearance of the superconductivity and the formation of $U$ magnetic moments and their ferromagnetic order. Namely, $\mathrm{UH}_{3-}$ $\mathrm{Mo}_{0.18}$ [39] and $\left(\mathrm{UH}_{3}\right)_{1-x} \mathrm{Zr}_{x}$ [40] are amorphous $5 f$ ferromagnets with $T_{\mathrm{C}}$ around $200 \mathrm{~K}$, i.e., with the Curie temperature higher that of $\alpha-\mathrm{UH}_{3}$ and $\beta-\mathrm{UH}_{3}(175 \mathrm{~K}[1$, 41]). Preliminary data on other $\gamma-\mathrm{U}$ splats (U-15 at.\% Pt and $\mathrm{U}-15$ at. $\% \mathrm{Nb}$ ) indicate that the hydrogen resistance is reproduced as well.

\section{Conclusions}

The $\gamma-\mathrm{U}$ phase can be retained in the $\mathrm{U}-T$ alloys by a combination of ultrafast cooling and doping with 15 at.\% $T$ content $(T=\mathrm{Mo}, \mathrm{Pt}, \mathrm{Nb})$ and 30 at. $\% \mathrm{Zr}$ content. $\mathrm{An}$ ideal $b c c$ A2 structure was found only in the U-15 at.\% Mo splat. Our results confirm that Mo is the best alloying 
element for stabilization of the $\gamma$-U structure. It is crucial that using ultrafast cooling we are able to reduce the necessary concentration of the $T$ elements $(T=\mathrm{Mo}, \mathrm{Nb}, \mathrm{Zr})$; i.e., the $\gamma$-U phase can be stabilized by a lower concentration of alloying elements. On the other hand, ultrafast cooling could also extend a higher solubility of Pt and Pd metals in $\gamma-\mathrm{U}$ and retain it to low temperatures. We emphasize again that all splat-cooled alloys were obtained without any additional treatment and they are very stable when exposing to ambient conditions.

All the $\mathrm{U}-T$ splats become superconducting with $T_{\mathrm{c}}$ in the range of $0.61-2.11 \mathrm{~K}$. The prediction of BCS superconductivity for the specific-heat jump at $T_{\mathrm{c}}$ was found to be fulfilled in the U-15 at.\% Mo among all investigated splats.

The U-T alloys with cubic $\gamma-\mathrm{U}$ phase reveal a high resistance against hydrogen absorption. The hydrogen absorption takes place only in a high hydrogen pressure $\left(\geq 2.5 \times 10^{5} \mathrm{~Pa}(2.5 \mathrm{bar})\right)$. Hydrogen can be easily desorbed by heating to $500-600{ }^{\circ} \mathrm{C}$ in vacuum. Thus, from the technological viewpoint, our study indicates an easy route for producing the $\gamma-U$ phase in a powder form: by a combination of hydrogenation, followed by crushing of the brittle hydrides and then hydrogen desorption in a vacuum.

Acknowledgments This work was supported by the Czech Science Foundation under the Grant No. 15-01100S. Experiments were partly performed at MLTL (http://mltl.eu/) supported within the program of Czech Research Infrastructures (No. LM2011025). M.P. was supported by the Grant Agency of the Charles University under the Project No. 1332314. Participation of Krakow group was supported by the Czech-Polish cooperation in the scope of Czech-Polish project 7AMB14PL036 (9004/R14/R15). N-T.H.K-N acknowledges the European Regional Development Fund under the Infrastructure and Environment Programme.

\section{References}

[1] I. Grenthe, J. Drozdzynski, T. Fujino, E.C. Buck, T.E. AlbrechtSchmitt, S.F. Wolf, in The Chemistry of the Actinide and Transactinide Elements, vol. 1, ed. by L.R. Morss, N. Edelstein, J. Fuger, J.J. Katz (Springer, 2006), p. 253

[2] H.L. Yakel, A review of X-ray diffraction studies in uranium alloys, in Proceedings of the Physical Metallurgy of Uranium Alloys Conference, Vail, Colorado, USA, 12-14 February 1974

[3] G. Aschermann, E. Justi, Phys. Z. 43, 207 (1942)

[4] G.H. Lander, E.S. Fisher, S.D. Bader, Adv. Phys. 43, 1 (1994)

[5] J.C. Lashley, J.C. Lashley, B.E. Lang, J. Boerio-Goates, B.F. Woodfield, G.M. Schmiedeshoff, E.C. Gay, C.C. McPheeters, D.J. Thoma, W.L. Hults, J.C. Cooley, R.J. Hanrahan Jr, J.L. Smith, Phys. Rev. B 63, 224510 (2001)

[6] D. Graf, R. Stillwell, T.P. Murphy, J.H. Park, M. Kano, E.C. Palm, P. Schlottmann, J. Bourg, K.N. Collar, J. Cooley, J. Lashley, J. Willit, S.W. Tozer, Phys. Rev. B 80, 241101R (2009)

[7] G.L. Hofman, M.K. Meyer, A.E. Ray, Design of high density gamma-phase uranium alloys for LEU dispersion fuel applications, in Proceedings of International Reduced Enrichment for
Research and Test Reactors Conference, Sao Paulo, Brazil, 18-20 October 1998

[8] V.P. Sinha, P.V. Hegde, G.J. Prasad, G.K. Dey, H.S. Kamath, J. Alloys Compd. 506, 253 (2010)

[9] S. Van Den Berghe, A. Leenaers, E. Koonen, L. Sannen, Adv. Sci. Technol. 73, 78 (2010)

[10] S. Van Den Berghe, P. Lemoine, Nucl. Eng. Technol. 46, 125 (2014)

[11] M.K. Meyer, G.L. Hofman, S.L. Hayes, C.R. Clark, T.C. Wiencek, J.L. Snelgrove, R.V. Strain, K.-H. Kim, J. Nucl. Mater. 304, 221 (2002)

[12] D.E. Burkes, R. Prabhakaran, T. Hartmann, J.-F. Jue, F.J. Rice, Nucl. Eng. Des. 240, 1332 (2010)

[13] J. Lisboa, J. Marin, M. Barrera, H. Pesenti, World J. Nucl. Sci. Technol. 5, 274 (2015)

[14] B.S. Chandrasekhar, J.K. Hulm, J. Phys. Chem. Solids 7, 259 (1958)

[15] T.G. Berlincourt, J. Phys. Chem. Solids 11, 12 (1959)

[16] H. Jones, Rep. Prog. Phys. 36, 1425 (1973)

[17] R. Ray, E. Musso, U.S. Patent 3,981,722, 21 Sept 1976

[18] I. Tkach, N.-T.H. Kim-Ngan, S. Mašková, M. Dzevenko, L. Havela, A. Warren, C. Stitt, T. Scott, J. Alloys Compd. 534, 101 (2012)

[19] N.-T.H. Kim-Ngan, I. Tkach, S. Maškova, A.P. Goncalves, L. Havela, J. Alloys Compd. 580, 223 (2013)

[20] G.C. Allen, P.M. Tucker, R.A. Lewis, J. Chem. Soc., Faraday Trans. II 80, 991 (1984)

[21] N.-T.H. Kim-Ngan, M. Paukov, S. Sowa, M. Krupska, I. Tkach, L. Havela, J. Alloys Compd. 645, 158 (2015)

[22] A. Dommann, F. Hulliger, Solid State Commun. 65, 1093 (1988)

[23] B.A.S. Ross, D.E. Peterson, Bull. Alloy Ph. Diagr. 11, 240 (1990)

[24] H. Kleykamp, Pure Appl. Chem. 63, 1401 (1991)

[25] K. Tangri, D.K. Chaudhuri, J. Nucl. Mat. 15, 278 (1965)

[26] M. Anagnostidis, M. Colombia, H. Monti, J. Nucl. Mat. 11, 67 (1964)

[27] S. Dash, K. Ghoshal, T.R.G. Kutty, J. Therm. Anal. Calorim. 112, 179 (2013)

[28] J.G. Huber, P.H. Ansari, Phys. B 135, 441 (1985)

[29] J.C. Slater, J. Chem. Phys. 41, 3199 (1964)

[30] I. Tkach, N.-T.H. Kim-Ngan, A. Warren, T. Scott, A.P. Goncalves, L. Havela, Physica C 498, 14 (2014)

[31] N.-T.H. Kim-Ngan, S. Sowa, M. Krupska, M. Paukov, I. Tkach, L. Havela, Adv. Nat. Sci. Nanosci. Nanotechnol. 6, 015007 (2015)

[32] N. Toyota, A. Inoue, K. Matsuzaki, T. Fukase, T. Masumoto, J. Phys. Soc. Jpn. 53, 924 (1984)

[33] A. Slebarski, J. Goraus, J. Deniszczyk, L. Skoczen, J. Phys.: Condens. Matter 18, 10319 (2006)

[34] A. Otop, I. Maksimov, E.-W. Scheidt, J.A. Mydosh, S. Sullow, Physica B 378-380, 371 (2006)

[35] J.S. Dugdale, Contemp. Phys. 28, 547 (1987)

[36] R.D. Barnard, Proc. Phys. Soc. 78, 722 (1961)

[37] L.E. DeLong, J.G. Huber, K.N. Yang, M.B. Maple, Phys. Rev. Lett. 51, 312 (1983)

[38] O. Pena, Physica C 514, 95 (2015)

[39] I. Tkach, S. Maskova, Z. Matej, N.-T.H. Kim-Ngan, A.V. Andreev, L. Havela, Phys. Rev. B 88, 060407R (2013)

[40] I. Tkach, M. Paukov, D. Drozdenko, M. Cieslar, B. Vondrackova, Z. Matej, D. Kriegner, A.V. Andreev, N.-T.H. Kim-Ngan, I. Turek, M. Divis, L. Havela, Phys. Rev. B 91, 115116 (2015)

[41] A.V. Andreev, S.M. Zadvorkin, M.I. Bartashevich, T. Goto, J. Kamarad, Z. Arnold, H. Drulis, J. Alloys Compd. 267, 32 (1998) 